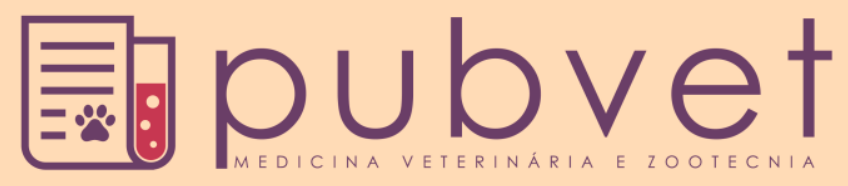

https://doi.org/10.22256/pubvet.v12n3a58.1-10

\title{
Uso de medicamentos homeopáticos para o tratamento da mastite bovina: Revisão
}

\author{
Renan Almeida de Jesus ${ }^{\bullet}$ 1*, César Alberto Coutinho² $^{2}$ \\ ${ }^{1 *}$ Farmacêutico, Departamento de Homeopatia, Nutriphós, Umuarama-PR, Brasil-E-mail: renanaj1988@gmail.com \\ ${ }^{2}$ Médico Veterinário, Departamento Técnico, Nutriphós, Umuarama-PR, Brasil-E-mail: cesarcoutinho@nutriphos.com.br \\ *Autor para correspondência
}

\begin{abstract}
RESUMO. A mastite se apresenta como a doença de maior importância em vacas de leite, devido aos prejuízos causados pela perda de produtividade e descarte de leite de acordo com alguns tratamentos, como os antimicrobianos. Diante da problemática dos antibióticos pela má aplicação ou resistência bacteriana, uma alternativa para o controle da mastite é o uso da homeopatia. $\mathrm{O}$ objetivo do presente trabalho é informar o que já foi pesquisado sobre o uso de medicamentos homeopáticos para o tratamento da mastite. A terapêutica homeopática se enquadra nos preceitos da pecuária orgânica, sendo um método adequado para essa finalidade. Os remédios utilizados para o tratamento da doença são das mais variadas origens, de plantas, minerais, animais e de grande representatividade, de isolados ou compostos bacterianos, de microrganismos que são causadores da mastite. A facilidade de administração dos medicamentos homeopáticos se apresenta como uma grande vantagem, pois na forma mais comum de uso, o mesmo pode ser adicionado na água de bebida ou misturado na ração evitando estresse por parte do animal. Os resultados na mastite devido à aplicação da homeopatia podem ser visualizados na variação da contagem de células somáticas, reações no teste CMT (Califórnia Mastite Teste), ausência de isolamento de microrganismos do leite e diminuição da porcentagem de casos de mastite clínica e subclínica. Embora o tratamento homeopático se mostre uma ótima alternativa para o controle e tratamento da mastite, os resultados são bastante variáveis devido às diferentes metodologias empregadas e o grande número de medicamentos homeopáticos diferentes utilizados.
\end{abstract}

Palavras chave: homeopatia, mastite, medicina alternativa, tratamento, vacas de leite

\section{Use of homeopathic medicines for the treatment of bovine mastitis: Review}

\begin{abstract}
Mastitis presents as the most important disease in milk cows, due to the damages caused by loss of productivity and milk discharge according to some treatments, such as antimicrobials. Faced with the problem of antibiotics due to poor application or bacterial resistance, an alternative for the control of mastitis is the use of homeopathy. The aim of the present study is to inform what has already been researched about the use of homeopathic medicines for the treatment of mastitis. Homeopathic therapy fits within the precepts of organic farming, being a suitable method for this purpose. The remedies used for the treatment of the disease are from the most diverse origins, plants, minerals, animals and of great representatively, of isolates or bacterial compounds, of microorganisms that are the cause of mastitis.
\end{abstract}


The ease of administration of homeopathic medicines presents a great advantage, since in the most common form of use, it can be added in drinking water or mixed in the feed avoiding stress on the part of the animal. The results in mastitis due to the application of homeopathy can be visualized in the variation of the somatic cell count, reactions in the CMT (California Mastitis Test), absence of isolation of milk microorganisms and decrease in the percentage of cases of clinical and subclinical mastitis. Although the homeopathic treatment proves to be a great alternative for the control and treatment of mastitis, the results are quite variable due to the different methodologies used and the large number of different homeopathic medicines used.

Keywords: alternative medicine, homeopathy, mastitis, milk cows, treatment

\section{Uso de medicamentos homeopáticos para el tratamiento de la mastitis bovina: Revisión}

RESUMEN. La mastitis se presenta como la enfermedad de mayor importancia en vacas de leche, debido a los daños causados por la pérdida de productividad y descarte de leche de acuerdo con algunos tratamientos, como los antimicrobianos. Ante la problemática de los antibióticos por la mala aplicación o resistencia bacteriana, una alternativa para el control de la mastitis es el uso de la homeopatía. El objetivo del presente trabajo es informar lo que ya se ha investigado sobre el uso de medicamentos homeopáticos para el tratamiento de la mastitis. La terapéutica homeopática se encuadra en los preceptos de la ganadería orgánica, siendo un método adecuado para esa finalidad. Los medicamentos utilizados para el tratamiento de la enfermedad son de los más variados orígenes, de plantas, minerales, animales y de gran representatividad, de aislados o compuestos bacterianos, de microorganismos que son causantes de la mastitis. La facilidad de administración de los medicamentos homeopáticos se presenta como una gran ventaja, pues en la forma más común de uso, el mismo puede ser añadido en el agua de bebida o mezclado en la ración evitando estrés por parte del animal. Los resultados en la mastitis debido a la aplicación de la homeopatía se pueden ver en la variación del recuento de células somáticas, reacciones en la prueba CMP (California Mastitis Prueba), ausencia de aislamiento de microorganismos de la leche y disminución del porcentaje de casos de mastitis clínica y subclínica. Aunque el tratamiento homeopático se muestra una óptima alternativa para el control y tratamiento de la mastitis, los resultados son bastante variables debido a las diferentes metodologías empleadas y el gran número de medicamentos homeopáticos diferentes utilizados.

Palabras clave: homeopatía, mastitis, medicina alternativa, tratamiento, vacas de leche

\section{Introdução}

A mastite é considerada o maior problema quando se refere aos bovinos leiteiros, mesmo apresentando várias formas de tratamento (Varshney \& Naresh, 2005). A doença é considerada de suma importância devido às perdas econômicas pela menor produtividade $\mathrm{e}$ as perdas no descarte de leite quando usados certos tipos de tratamento (Halasa et al., 2009).

A medida mais comum no controle da mastite é o tratamento com antimicrobianos (Almeida et al., 1999), mas o tratamento com antibióticos apresenta problemas de resistência microbiana quando utilizado de forma descontrolada (Ganda et al., 2016). Na busca pela redução do uso desses fármacos, a homeopatia se apresenta como alternativa para o tratamento de doenças presentes no rebanho, como a mastite (Doehring \& Sundrum, 2016). Além de contemplar o bem-estar animal, a homeopatia pode ser considerada uma alternativa em função de seu menor custo e sua melhor administração (uma vez que o medicamento pode ser fornecido na água, ração ou sal mineral) (Costa et al., 2009). A falta de informações sobre os medicamentos homeopáticos e seu tratamento contribui para o 
não uso da homeopatia (Honorato et al., 2007), e diante dessa problemática, o objetivo do presente trabalho é apresentar o que tem sido realizado no uso do tratamento homeopático na mastite bovina.

\section{Mastite}

A mastite é um resultado comum de uma infecção da glândula mamária, geralmente de causa bacteriana, sendo a doença de maior importância nos bovinos leiteiros (Wellenberg et al., 2002, Herry et al., 2017) sendo classificada como clínica ou subclínica. O diagnóstico é realizado por meio de exame clínico, além de exames complementares como teste de tamis e Califórnia Mastite Teste (CMT) (Peixoto et al., 2009), sendo o clínico de fácil visualização da mastite clínica; enquanto que o CMT proporciona diagnosticar também a mastite subclínica. Costa et al. (2017) encontraram que pesquisas definem como vacas sadias aquelas com contagem de células somáticas (CCS) menores que 200.000 células $\mathrm{mL}^{-1}$ e vacas com mastite subclínica aquelas com CCS maiores que 200.000 células $\mathrm{mL}^{-1}$.

A mastite subclínica acarreta prejuízos econômicos significativos ao produtor, em função da diminuição de produção, da qualidade do leite, aumento dos custos com tratamento e descarte de animais e do leite dos animais tratados (Wellenberg et al., 2002, Halasa et al., 2009, Costa et al., 2017). Devido aos prejuízos econômicos, o uso de métodos de controle contra a mastite se tornam necessários para rebanhos que apresentem a doença (Zafalon et al., 2017a, Zafalon et al., 2017b).

A etiologia da mastite se apresenta como multifatorial, onde diversos fatores podem afetar direto ou indiretamente a presença da enfermidade, tornando-se um desafio a prevenção e tratamento nos animais afetados (Wellenberg et al., 2002, Ganda et al., 2016). Pode-se citar como fatores predisponentes da doença, o clima, as instalações e a cama onde ficam os animais (pois podem agir como fontes de contaminação microbiana), o estresse submetido ao rebanho, genética, fatores nutricionais e fatores humanos relacionados com o manejo (Pires et al., 2004, Zafalon et al., 2017a, Zafalon et al., 2017b).

Algumas medidas preventivas da mastite são a adequação do ambiente, manejo correto de dejetos, correta preparação do úbere na ordenha, com realização de pré e pós-dipping, separação de animais com mastite de maior severidade, linha de ordenha com animais com animais sadios sendo manejados primeiro, adequada manutenção e higienização dos equipamentos utilizados na ordenha, manter animais em pé após ordenha e alimentação adequada para o rebanho (Pires et al., 2004). Zafalon et al., (2017b) ainda cita a importância de medidas conjuntas no tratamento da mastite subclínica em ovelhas, medidas que podem ser utilizadas também no tratamento de bovinos.

De acordo com Santana et al. (2016) o processo inflamatório na glândula mamária desencadeado em resposta a um microrganismo invasor pode ser efetivo no combate à infecção, mas como esse efeito não é o suficiente para todos os casos, a medida mais comum no controle da mastite é o tratamento com antimicrobianos (Almeida et al., 1999).

\section{Uso de antimicrobianos}

$\mathrm{O}$ uso indiscriminado de antimicrobianos sem o conhecimento da suscetibilidade desses agentes patogênicos, atualmente resulta no crescimento da resistência bacteriana, o que pode ocasionar falhas no tratamento (Varshney \& Naresh, 2005, Girardini et al., 2016). O uso da penicilina e seus derivados, por exemplo, deve ser realizado apenas para o tratamento de mastites causadas por bactérias gram-positivas (Cazoto et al., 2011), informações que nem sempre estão disponíveis ao produtor e clínico.

Deve-se considerar o custo benefício do tratamento antibiótico, já que a utilização desses fármacos acarreta altos custos, além da presença de resíduos no leite, que é um problema de saúde pública e tecnológico em toda a cadeia leiteira (Almeida et al., 2011). O uso de antimicrobianos tem impacto direto na saúde, e aproximadamente $80 \%$ desses medicamentos prescritos para o uso em vacas de leite são usados apenas para tratar casos de mastite (Ganda et al., 2016).

A dose de antimicrobianos normalmente utilizada para o tratamento de infecções é mais elevada que a dose necessária para inativar os organismos patogênicos, o que favorece o aparecimento de efeitos indesejados (Saha et al., 2007), como intoxicação animal, destruição da microbiota natural e favorecimento de 
resistência microbiana, que ocasiona um diferente cenário epidemiológico, mais grave (Girardini et al., 2016). Novos compostos antibacterianos são investigados para utilização em infecções (Cazoto et al., 2011).

O impacto dos antibióticos de amplo espectro na microbiota do leite (além dos principais agentes patogênicos) permanece desconhecido. As preocupações relacionadas à segurança alimentar e ao desenvolvimento de resistência podem levar a uma diminuição da disponibilidade de antibióticos, e assim limitar a capacidade de controle da doença (Ganda et al., 2016). Diante dos efeitos adversos dessa terapia no tratamento da mastite, do bem-estar animal e produtividade desses rebanhos, é preciso avaliar a viabilidade de tratamentos alternativos (Peixoto et al., 2009).

É crescente a demanda por terapias alternativas (como fitoterapia e homeopatia) no tratamento de mastites e outras doenças que afetam o gado de leite. Esta procura está vinculada à limitação dos tratamentos convencionais e a busca aos sistemas de produção especializados, tais como produtos orgânicos (Pires et al., 2004).

\section{Pecuária orgânica}

O processo de certificação orgânica está cada vez mais rígido, e os diferentes países têm padrões diferentes em relação às práticas de produção orgânica, que variam enormemente em relação à aceitabilidade das substâncias utilizadas para a gestão da saúde. O manejo de doenças infecciosas, como a mastite, é alterado para cumprir estes regulamentos orgânicos, e o impacto dessas regulamentações sobre a saúde animal não foi bem documentado (Ruegg, 2009). O efeito da fitoterapia tem sido testado no combate aos patógenos da mastite, além dos estudos com medicamentos homeopáticos (Peixoto et al., 2009, Peixoto et al., 2016).

Devido ao crescimento da pecuária orgânica, a homeopatia tem sido cada vez mais utilizada, o que a insere em um setor de grande importância econômica para o país. Ruegg (2009) encontrou em sua revisão que a homeopatia representa o tratamento de escolha em mais da metade das propriedades com produção orgânica e sustentável. Representativa na produção do leite orgânico e também do leite convencional, pode ser considerada um caminho na busca da redução de custos de tratamento de doenças, reduzindo também os perigos à saúde pública (Thomaz et al., 2008, Costa et al., 2009).

O preço pago pelo leite orgânico é geralmente maior e mais estável. Pequenos produtores podem considerar a conversão para o status orgânico como uma forma de manter a lucratividade. No entanto, fatores que não são específicos da produção orgânica, como idade, nível de produção, genética, condições ambientais, nutrição e habitação podem influenciar a saúde animal e consequentemente sua produtividade (Ruegg, 2009).

\section{Homeopatia}

As propriedades terapêuticas dos medicamentos homeopáticos ganham cada vez mais espaço no tratamento veterinário (Almeida et al., 1999). Foram inicialmente utilizados na Alemanha em 1796, pelo médico Christian Friedrich Samuel Hahnemann e ganharam popularidade no tratamento de doenças humanas, sendo notificado seu uso na medicina veterinária apenas em 1833 com o profissional Johann Joseph Wilhelm Lux (Ruegg, 2009, Carneiro et al., 2011).

Os princípios e as leis da homeopatia veterinária são os mesmos da homeopatia humana e sua utilização é parecida. O tratamento é baseado no princípio ou "lei dos semelhantes", o qual envolve o tratamento da doença ou do sintoma com pequena quantidade de compostos que causam sintomas semelhantes aos da doença quando administrado em altas concentrações (Pires et al., 2004). Prescreve-se um medicamento homeopático a um animal doente em função de suas características e sintomas individuais (Pires et al., 2004), mas observam-se estudos feitos para validar sua aplicação no tratamento de grupo (Elliott, 2001).

Não existem problemas de resíduo, sem período de retirada do produto e sem poluição ambiental. Além disso, os remédios são relativamente baratos (Løken, 2002).

Têm sido propostas inúmeras teorias sobre o mecanismo de ação dos tratamentos homeopáticos, mas como a homeopatia induz o efeito terapêutico ainda é desconhecido, e o foco da maioria das pesquisas é identificar os efeitos dos tratamentos (Løken, 2002, Pires et al., 2004). Existem poucos dados de eficácia da homeopatia veterinária nos trabalhos de âmbito 
científico (Ruegg, 2009, Doehring \& Sundrum, 2016). O tratamento baseia-se na resposta do organismo contra a um estímulo externo, produzindo reações, e sabe-se que o processo de cura de um medicamento homeopático é diferente do convencional (chamado também de alopático), e está vinculado à imunologia, pois pode causar imunização indireta e inespecífica (Almeida et al., 1999, Almeida et al., 2011, Carneiro et al., 2011).

$\mathrm{Na}$ introdução da homeopatia como terapêutica, é importante que o produtor conheça o máximo possível sobre a mesma, pois uma baixa adesão pode residir no fato de não se conhecer as técnicas utilizadas, os objetivos e resultados pretendidos (Pires et al., 2004, Honorato et al., 2007). Com maior conhecimento sobre a ciência, a homeopatia ganha mais adeptos a essa abordagem dos cuidados de saúde animal (Searcy et al., 1995).

\section{Medicamentos utilizados}

O princípio ativo do medicamento homeopático não é utilizado em sua forma natural, mas sim dinamizados (diluições seguidas de triturações para medicamentos insolúveis e no caso de medicamentos solúveis, sucussões, ou seja, agitação vigorosa), com a finalidade de liberar a energia do medicamento, que é particular a cada princípio ativo e diferentes dinamizações ou potências (Costa et al., 2009, ANVISA, 2010).

Os medicamentos devem apresentar em seu rótulo, códigos para informar a dinamização e a técnica ou metodologia utilizada em sua preparação. $\mathrm{O}$ número indica o número de diluições que foram realizadas, e a letra indica a escala dessa diluição, onde $\mathrm{X}, \mathrm{D}$ ou DH são para diluições $1 / 10$ e $\mathrm{C}$ ou $\mathrm{CH}$ são para diluições 1/100, dentre outras escalas existentes (ANVISA, 2010). Por exemplo, um medicamento $6 \mathrm{DH}$ foi diluído seis vezes na escala decimal, enquanto que um medicamento $12 \mathrm{CH}$ foi diluído doze vezes na escala centesimal.

Duas formas de uso de medicamentos são observadas na prática homeopática veterinária, utilizando apenas um princípio ativo (chamado também de homeopatia individual ou clássica) ou medicamentos contendo dois ou mais princípios ativos (chamado de homeopatia complexa ou populacional) (Hektoen et al., 2004, Doehring \& Sundrum, 2016, Zafalon et al., 2017a, Zafalon et al., 2017b). Werner et al. (2010) relatam que na medicina veterinária, observa-se na maioria dos casos a preferência por utilização de potências ou dinamizações baixas, como DH6 ou DH12. Essa preferência pode estar relacionada com a maior presença do princípio ativo inicial. Sabe-se que a partir da $12 \mathrm{CH}$ não se encontram moléculas do princípio ativo que foi utilizado para preparar o medicamento (Costa et al., 2009, ANVISA, 2010), embora o medicamento continue sendo capaz de causar reações no organismo, inclusive obtendo a melhora das doenças como a mastite. No decorrer do tratamento, os sintomas relevantes podem mudar e, em seguida, correlacionados com os sintomas de outro remédio (Hektoen et al., 2004, Werner et al., 2010).

Os medicamentos de origem vegetal são geralmente os mais abundantes na prática homeopática, podendo ser utilizada a planta inteira, suas partes ou seus produtos extrativos (ANVISA, 2010). Os medicamentos utilizados de origem vegetal que foram encontrados em trabalhos para o tratamento de mastite foram Aconitum napellus (na potência $6 \mathrm{CH}$ ), Arnica montana (30CH) Asa foetida (6DH), Atropa belladona (30DH, $12 \mathrm{CH}$ e $30 \mathrm{CH})$, Bryonia alba (30DH e 30CH), Calendula officinalis (30DH), Conium maculatum (30CH e 200CH) Ipecacuanha $(30 \mathrm{CH})$, Phytolacca decandra (12DH, 30DH, $6 \mathrm{CH}, 12 \mathrm{CH}, 30 \mathrm{CH}$ e $200 \mathrm{CH}$ ), Pulsatilla nigricans $(6 \mathrm{DH}, 30 \mathrm{DH}, 6 \mathrm{CH}$ e $30 \mathrm{CH})$, Urtica urens (30DH e 30CH) (Searcy et al., 1995, Varshney \& Naresh, 2005, Santos \& Griebeler, 2006, Martins et al., 2007, Klocke et al., 2010, Werner et al., 2010, Leal \& Costa, 2013, Almeida et al., 2015, Zafalon et al., 2017a, Zafalon et al., 2017b). As indicações dos medicamentos citados podem ser encontradas na literatura, Aconitum napellus, por exemplo, é indicado para febres e estados inflamatórios agudos, assim como a Calendula officinalis, que atua como anti-inflamatório e Urtica urens, medicamento utilizado para o aumento da produção de leite (ANVISA, $\underline{2010)}$.

Já para os medicamentos de origem animal, podem ser oriundos do animal inteiro (como abelhas, formigas), suas partes e produtos extrativos, patológicos ou não (ANVISA, 2010). Para esta classe foram encontrados Apis mellifica (não foi descrita a dinamização 
utilizada), Calcarea carbonica (nas dinamizações 6DH e 6CH), Carbo animalis

$(12 \mathrm{CH})$, Lachesis muta (6DH e 12DH) e Sepia succus (6DH) (Martins et al., 2007, Klocke et al., 2010, Werner et al., 2010, Leal \& Costa, 2013, Almeida et al., 2015). Por exemplo, o medicamento Apis mellifica é indicado para doenças que apresentam edema e/ou inflamação aguda ( acontece na mastite.

Os medicamentos de origem mineral podem ser obtidos em sua forma natural, extraídos, purificados e produzidos por indústrias farmacêuticas, bem como misturas químicas padronizadas por Hahnemann (ANVISA, 2010). Os medicamentos de origem mineral ou química encontrados em experimentos para tratamento de mastite foram Calcarea fluorica (200CH), Calcium phosphoricum (6DH), Hepar sulphur (200DH, 12CH e 30CH), Kalium muriatium (6DH), Lac vaccinum $(12 \mathrm{CH})$, Magnesium fluoricum (200DH e $12 \mathrm{CH})$, Mercurius solubilis $(6 \mathrm{DH}, 6 \mathrm{CH}$ e $30 \mathrm{CH})$, Phosphorus albus $(12 \mathrm{CH}, 30 \mathrm{CH}$ e $200 \mathrm{CH})$, Silicea terra $(6 \mathrm{DH}, 30 \mathrm{DH}, 6 \mathrm{CH}$, $12 \mathrm{CH}$ e $30 \mathrm{CH}$ ), Sulphur (6DH, 30DH e 12CH) (Searcy et al., 1995, Varshney \& Naresh, 2005, Santos \& Griebeler, 2006, Martins et al., 2007, Barzon et al., 2008, Thomaz et al., 2008, Klocke et al., 2010, Werner et al., 2010, Leal \& Costa, 2013, Almeida et al., 2015, Zafalon et al., 2017a, Zafalon et al., 2017b). Os medicamentos Mercurius solubilis $\mathrm{e}$ Phosphorus albus, por exemplo, são indicados para qualquer tipo de inflamação, sendo que o segundo também para fissuras, sintomas facilmente enquadrados em casos de mastite (Costa et al., 2009).

Dentre os medicamentos homeopáticos, os isoterápicos ou bioterápicos, ou seja, medicamentos elaborados a partir de excreções e secreções, alérgenos, produtos de origem microbiana e órgãos e tecidos, vem sendo utilizados com frequência (ANVISA, 2010). Os nosódios utilizados para o tratamento da mastite são baseados em extração dos organismos etiológicos de amostras de leite contaminado e são recomendados para reduzir a severidade, duração e número de casos (Pires et al., 2004).

Os medicamentos bioterápicos encontrados foram o bioterápico de isolado bacteriano (na potência $12 \mathrm{CH}$ ), bioterápico e nosódios
Staphylococcinum (200DH, 12CH), Streptococcinum (200DH), bioterápico complexo (na dinamização $30 \mathrm{CH}$ ), nosódio complexo (Streptococcus uberis, Streptococcus dysgalactiae, Streptococcus agalactiae, Escherichia coli e Staphylococcus aureus, sem informar dinamização e outro com $30 \mathrm{CH}$ ) e isoterápico feito do leite (Day, 1986, Almeida et al., 1999, Morales et al., 2005, Martins et al., 2007, Barzon et al., 2008, Thomaz et al., 2008, Almeida et al., 2011, Kiarazm et al., 2011).

\section{Formas de aplicação}

A literatura sobre o assunto é bastante escassa, os protocolos de tratamento e metodologia são bastante variáveis (Almeida et al., 1999).

O ideal é que seja realizada uma avaliação individual dos animais para se avaliar a maior ou menor presença de mastite no rebanho. Além da avaliação clínica das glândulas mamárias, é de grande importância a utilização de testes, como o controle microbiológico do leite, CCS e CMT (Honorato et al., 2007, Almeida et al., 2015) para definir os grupos de tratamento e administrar diferentes doses de medicamento de acordo com a gravidade. Barzon et al. (2008) dividiram as vacas em 3 grupos de acordo com o resultado do CMT para facilitar o manejo e aplicação dos tratamentos.

Os remédios podem ser preparados para que fiquem palatáveis, facilitando sua administração oral, mas podem ser aplicados também localmente, pelas mucosas ocular e vaginal. Há também a facilidade de se ministrar doses únicas ou doses diárias na alimentação, assim evita-se o estresse ocasionado pela administração forçada, uso de seringas e demais manobras dolorosas (Pires et al., 2004).

A dosagem, frequência e duração do tratamento são variáveis. Para tratamento de todo o rebanho, a administração é majoritariamente oral (via água de bebida ou pó, como açúcar e sal mineral); enquanto que para o tratamento individual os remédios homeopáticos podem ser administrados oralmente (via glóbulos, gotas ou pó), ou ainda de forma mais rara, por via subcutânea, injeção intramuscular, tópica (via mucosa vaginal, úbere) ou por injeção intramamária (Almeida et al., 1999, Hektoen et al., 2004, Pires et al., 
2004, Varshney \& Naresh, 2005, Doehring \& Sundrum, 2016).

\section{Resultados esperados com o uso da homeopatia}

Compreender os fatores que contribuem para a adoção ou rejeição da homeopatia, especialmente no período de transição de sistemas, pode colaborar com o sucesso de programas que visam à difusão dessa terapêutica (Honorato et al., 2007). A exclusão da mastite causada por agentes patogénicos associados ao úbere no rebanho leiteiro em combinação com um procedimento melhorado de diagnóstico é importante quando se usa a estratégia de tratamento homeopático (Werner et al., 2010), possibilitando melhores resultados.

A literatura descreve resultados variáveis em relação ao tratamento de bovinos com mastites usando diferentes preparações, vias e dosagens. A comparação de resultados do tratamento com medicamentos homeopáticos entre produtos convencionais tem a problemática de que a metodologia adotada pode ter sido diferente (Almeida et al., 2005). De acordo com Day (1986), a comparação dos resultados para os tratamentos homeopáticos deve ser realizada de maneira sistemática, pois a falta de dados ou mesmo a falta de clareza na descrição da metodologia e critérios adotados podem limitar as conclusões. Em parte, a dificuldade dos pesquisadores é que os mesmos utilizam os procedimentos para testes de medicamentos convencionais, que pode não ser o melhor método para o entendimento da farmacologia homeopática (Thomaz et al., 2008). Os parâmetros para cura variam e baseiam-se geralmente na ausência de isolamentos de microrganismos no leite, respostas de CMT e CCS em mastites clínicas e subclínicas (Werner et al., 2010, Almeida et al., 2011). No experimento realizado por Martins et al. (2007) observou-se redução na frequência de mastite subclínica desde o primeiro mês de tratamento, sendo que a mesma continuou em declínio até o período de 90 dias, que foi o período de experimentação, demonstrando a possibilidade da utilização da homeopatia com resultados rápidos sem perda na produção leiteira. Doehring \& Sundrum (2016) concluíram em uma revisão sobre o assunto, que a terapêutica homeopática é efetiva contra a mastite, quando comparada à grupos controle, resultados que foram observados também por Almeida et al. (2005). Doehring \& Sundrum (2016) ainda observaram que as taxas de cura dos medicamentos homeopáticos variavam entre 5 e $80 \%$; enquanto que as taxas de cura com o tratamento convencional antimicrobiano tinha resultados variáveis entre 17 e 95\%. Almeida et al. (2011) chegaram ao resultado de ausência de isolamento microbiano de Staphylococcus aureus em $60 \%$ dos tetos tratados; enquanto que a ausência no grupo controle foi de apenas $32,6 \%$. Em outro trabalho, Almeida et al. $\underline{(2005)}$ verificaram cura microbiológica em $72,7 \%$ dos tetos tratados. Kiarazm et al. (2011) concluíram que o uso de medicamentos homeopáticos reduziu o isolamento de bactérias no leite e também ocasionaram uma diminuição na CCS, resultados que corroboram para a diminuição da mastite. Resultados de diminuição na contagem de células somáticas também foram encontrados por Barzon et al. (2008) em 82\% dos animais tratados. Martins et al. (2007) constataram em seu experimento que houve redução na mastite subclínica de $44,5 \%$ para $3,9 \%$ no rebanho, sendo que o uso do medicamento homeopático foi a única prática de manejo alterada na propriedade. Os resultados relatados demonstram que há uma resposta do organismo quando se administra um medicamento homeopático.

De acordo com Almeida et al. (1999), mesmo diante de bons resultados, é necessária a otimização do tratamento, pois pela diversidade etiológica e alta prevalência da doença, tem-se o pressuposto de que se utilizado em todo o rebanho, seja com a finalidade de cura ou prevenção, os resultados sejam ainda melhores. Mesmo com evidências de trabalhos com resultados positivos com o tratamento homeopático, as informações ainda são limitadas para justificar uma conclusão definitiva (Pires et al., 2004), sendo necessário o desenvolvimento de outros experimentos por períodos diferentes, como em duas estações diferentes do ano, número maior de animais, manejos diferenciados e dosagens divergentes para observar se os resultados são eficazes ou não (Leal \& Costa, 2013), levando ainda em consideração os diferentes medicamentos que podem ser utilizados.

Não foram encontrados exclusivamente trabalhos com bons resultados do tratamento 
homeopático para a cura da mastite. Santos Júnior et al. (2010) concluíram que o uso de homeopatia não alterou os valores de CMT e CCS, tampouco a produção leiteira. Porém quando avaliaram a média máxima de contagem de células somáticas, verificou-se melhora nos quadros clínicos. Resultados não significativos para CCS também foram relatados por Thomaz et al. (2008), cujo autor levantou a hipótese que em uma tentativa do organismo de restabelecer o equilíbrio, a homeopatia pode ter promovido o estado de proteção da glândula mamária.

O tempo de resposta do organismo frente a um medicamento homeopático é proporcional ao tempo da afecção, portanto, diante de um processo agudo tem-se a resposta em poucas horas (Pires et al., 2004), contrariando o conhecimento popular de que a homeopatia só tem resultados em longo prazo.

Sobre o custo, o produto homeopático por ser de uso oral independe da quantidade de úberes afetados pela doença (Santos \& Griebeler, 2006), diferente de alguns tratamentos convencionais de aplicação intramamária, o que faz que o tratamento homeopático seja mais barato.

No trabalho de Werner et al. (2010), os animais que não obtiveram respostas ao tratamento homeopático foram posteriormente tratados com antibióticos, enquanto os não respondedores aos antimicrobianos foram submetidos à terapêutica homeopática, demonstrando que os dois sistemas de tratamento podem ser utilizados, e que ambos apresentam tanto falhas, quanto vantagens.

\section{Conclusão}

Conclui-se que o tratamento homeopático pode ser utilizado como alternativa aos antimicrobianos para o tratamento da mastite em bovinos de leite, com resultados que podem aparecer na contagem de células somáticas, isolamento de microrganismos do leite, e diferentes níveis de reações no CMT (Califórnia Mastite Teste).

Novos experimentos com medicamentos homeopáticos devem ser realizados com comparação com grupos controle para elucidar a resposta das vacas frente aos variados medicamentos que podem ser utilizados e as diferentes vias de aplicação.

\section{Referências bibliográficas}

Almeida, A. C., Fonseca, Y. M., Soares, T. M. P., Silva, D. B., Buelta, T. T. M. \& Silva, G. L. M. E. 1999. Tratamento de mastite subclínica em bovinos utilizando bioterapia. Revista da Universidade de Alfenas, 5, 199-203.

Almeida, A. C., Soares, T. M. P., Silva, D. B., Silva, B. C. M., Almeida, P. N. M. \& Santos, C. A. 2011. Atividade de bioterápicos para o tratamento de mastite subclínica bovina. Revista Brasileira de Agroecologia, 6, 134-141.

Almeida, A. C., Soares, T. M. P., Silva, D. B., Silveira, A. d. L., Fiorini, J. E. \& Fonseca, Y. M. 2005. Eficácia de tratamento homeopático no controle da mastite subclinica em bovinos. Veterinária Notícias, 11, 53-59.

Almeida, L. A. B., Brito, M. A. V. P., Brito, J. R. F., Pires, F. A. \& Benites, N. R. 2015. Tratamento de mastite clínica experimental por meio de ordenhas múltiplas em vacas leiteiras inoculadas com Staphylococcus aureus. Arquivo Instituto Biológico, 72, 16.

ANVISA. 2010. Farmacopeia Brasileira, 5 edn. Agência Nacional de Vigilância Sanitária e Fundação Oswaldo Cruz, Brasília.

Barzon, C. D., Takemura, O. S., Silva, L. M., Massambani, C., Moraes, R. E., Medeiros, F. \& Gazim, Z. C. 2008. Preliminary study of homeopathic treatment of subclinical mastitis evaluated through somatic cells count (SCC) and California mastitis test (CMT). International Journal of High Dilution Research, 7, 147-151.

Carneiro, S. M. T. P., Teixeira, M. Z., Nechar, R. M. C., Lonni, A. A., Rodrigues, M. R. \& Filippsen, L. 2011. Homeopatia: princípios $e$ aplicações na agroecologia. IAPAR, Londrina, Paraná.

Cazoto, L. L., Martins, D., Ribeiro, M. G., Durán, N. \& Nakazato, G. 2011. Antibacterial activity of violacein against Staphylococcus aureus isolated from bovine mastitis. The Journal of Antibiotics, 64, 395-397.

Costa, H. N., Molina, L. R., Lage, C. F. A., Malacco, V. M. R., Facury Filho, E. J. \& Carvalho, A. Ú. 2017. Estimativa das 
perdas de produção leiteira em vacas mestiças Holandês x Zebu com mastite subclínica baseada em duas metodologias de análise. Arquivo Brasileiro de Medicina Veterinária e Zootecnia, 69, 579-586.

Costa, N. C., Araújo, R. L. \& Freitas, G. B. L. 2009. Homeopatia: Um campo terapêutico fundamental no cuidado veterinário de animais de produção. Revista Salus, 3, 7389.

Day, C. 1986. Clinical trials in bovine mastitis: use of nosodes for prevention. British Homoeopathic Journal, 75, 11-14.

Doehring, C. \& Sundrum, A. 2016. Efficacy of homeopathy in livestock according to peerreviewed publications from 1981 to 2014 . The Veterinary Record, 179, 628.

Elliott, M. 2001. Cushing's Disease: a new approach to therapy in equine and canine patients. British Homoeopathic Journal, 90, 33-36.

Ganda, E. K., Bisinotto, R. S., Lima, S. F., Kronauer, K., Decter, D. H., Oikonomou, G., Schukken, Y. H. \& Bicalho, R. C. 2016. Longitudinal metagenomic profiling of bovine milk to assess the impact of intramammary treatment using a thirdgeneration cephalosporin. Scientific Reports, 6, 1-13.

Girardini, L. K., Paim, D. S., Ausani, T. C., Lopes, G. V., Pellegrini, D. C. P., Brito, M. A. V. P. \& Cardoso, M. 2016. Antimicrobial resistance profiles of Staphylococcus aureus clusters on small dairy farms in southern Brazil. Pesquisa Veterinária Brasileira, 36, 951-956.

Halasa, T., Nielen, M., De Roos, A. P. W., Van Hoorne, R., De Jong, G., Lam, T. J. G. M., Van Werven, T. \& Hogeveen, H. 2009. Production loss due to new subclinical mastitis in Dutch dairy cows estimated with a test-day model. Journal of Dairy Science, 92, 599-606.

Hektoen, L., Larsen, S., Ødegaard, S. A. \& Løken, T. 2004. Comparison of homeopathy, placebo and antibiotic treatment of clinical mastitis in dairy cowsmethodological Issues and results from a randomized- clinical trial. Transboundary and Emerging Diseases, 51, 439-446.

Herry, V., Gitton, C., Tabouret, G., Répérant, M., Forge, L., Tasca, C., Gilbert, F. B., Guitton, E., Barc, C. \& Staub, C. 2017.
Local immunization impacts the response of dairy cows to Escherichia coli mastitis. Scientific Reports, 7, 3441.

Honorato, L. A., Hötzel, M. J., Machado-Filho, L. C. P. \& Karam, K. F. 2007. The adoption of homeopathy in small Brazilian dairy farms. International Journal of High Dilution Research, 6, 22-26.

Kiarazm, M., Tajik, P. \& Nava, H. G. 2011. Assessment of the effect of homoeopathic nosodes in subclinical bovine mastitis. Annals of Biological Research, 2, 552-562.

Klocke, P., Ivemeyer, S., Butler, G., Maeschli, A. \& Heil, F. 2010. A randomized controlled trial to compare the use of homeopathy and internal Teat Sealers for the prevention of mastitis in organically farmed dairy cows during the dry period and 100 days post-calving. Homeopathy, 99, 90-98.

Leal, L. P. \& Costa, C. 2013. Utilização da homeopatia para redução da contagem de células somaticas (CCS) em vacas Girolando. PUBVET, 7, 1304-1321.

Løken, T. 2002. Alternative therapy of animals-homeopathy and other alternative methods of therapy. Acta Veterinaria Scandinavica, 43, 47-50.

Martins, C. R., Vieria, E. C., Gazim, Z. C. \& Massambani, C. 2007. Tratamento de mastite subclínica por meio de suplementação mineral homeopática da dieta de vacas leiteiras em lactação-estudo de caso. Cultura Homeopática, 19, 16-19.

Morales, R. V., Menéndez, C. C., Pasos, F. L., Quiñones, R. N. \& Cosío, E. C. 2005. Reylac, una alternativa homeopatica en el control de la mastitis subclinica bovina. Revista Eletrônica de Vetrinária, 6, 1-7.

Peixoto, E. C. T. M., Pelanda, A. G., Radis, A. C., Heinzen, E. L., Garcia, R. C. \& Valério, M. A. 2009. Incidência de mastite bovina em animais homeopatizados. Revista do Instituto de Laticínios Cândido Tostes, 64, 66-71.

Peixoto, R. D. M., Silva, W. E. L., Almeida, J. R. G. S., Branco, A. \& Costa, M. M. 2016. Antibacterial potential ff native plants from the caatinga biome against Staphylococcus spp. isolates from small ruminants with mastitis. Revista Caatinga, 29, 758-763. 
Pires, M., Brito, J. R. F. \& Brito, M. A. V. P. 2004. Homeopatia: uma opção de tratamento da mamite bovina. Embrapa Gado de Leite, 1, 1-39.

Ruegg, P. L. 2009. Management of mastitis on organic and conventional dairy farms. Journal of Animal Science, 87, 43-55.

Saha, B., Bhattacharya, J., Mukherjee, A., Ghosh, A., Santra, C., Dasgupta, A. K. \& Karmakar, P. 2007. In vitro structural and functional evaluation of gold nanoparticles conjugated antibiotics. Nanoscale Research Letters, 2, 614-622.

Santana, R. C. M., Zafalon, L. F., Brandão, H. M., Junior, G. A. F., Pilon, L. E., Junior, W. B., Giglioti, R. \& Mosqueira, V. C. F. 2016. Uso de antimicrobiano nanoparticulado para o tratamento da mastite subclínica de ovelhas de corte no período seco. Pesquisa Veterinária Brasileira, 36, 826-830.

Santos, J. \& Griebeler, S. A. 2006. Tratamento homeopático da mastite do gado leiteiro. Cultura Homeopática, 14, 11-13.

Santos Júnior, J. H. R., D'auria, E., Agostinho, J. M. A. \& Freitas, F. C. 2010. Avaliação do efeito do medicamento isoterápico comercial na prevenção de mastite subclínica. Nucleus Animalium, 2, 99-106.

Searcy, R., Reyes, O. \& Guajardo, G. 1995. Control of subclinical bovine mastitis: utilization of a homoeopathic combination. British Homoeopathic Journal, 84, 67-70.

Thomaz, L. W., Mesquita, A. J., Macedo, E. F., Godoy, R. R., Bueno, V. F. F. \& Nunes, R. d. C. 2008. Utilização de silicea $12 \mathrm{CH}$ e bioterápico complexo $30 \mathrm{CH}$ na contagem de células somáticas em vacas leiteiras.
Revista Científica de Produção Animal, 10, 138-149.

Varshney, J. P. \& Naresh, R. 2005. Comparative efficacy of homeopathic and allopathic systems of medicine in the management of clinical mastitis of Indian dairy cows. Homeopathy, 94, 81-85.

Wellenberg, G. J., Van der Poel, W. H. M. \& Van Oirschot, J. T. 2002. Viral infections and bovine mastitis: a review. Veterinary Microbiology, 88, 27-45.

Werner, C., Sobiraj, A. \& Sundrum, A. 2010. Efficacy of homeopathic and antibiotic treatment strategies in cases of mild and moderate bovine clinical mastitis. Journal of Dairy Research, 77, 460-467.

Zafalon, L. F., Alves, T. C. \& Chagas, A. C. S. 2017a. Uso de homeopatia para o controle de mastite subclínica bovina. Embrapa Pecuária Sudeste-Boletim de Pesquisa e Desenvolvimento, 1, 1-29.

Zafalon, L. F., Cunha, M. L. R. S., Souza, M. R., Riboli, D. F. M. \& Pilon, L. E. 2017b. Persistência de Staphylococcus coagulasenegativos em glândulas mamárias de ovelhas com mastite subclínica após o tratamento antimicrobiano à secagem. Ciência Animal Brasileira, 18, 1-11.

Article History:

Received 20 December 2017

Accepted 2 February 2018

Available online 1 March 2018

License information: This is an open-access article distributed under the terms of the Creative Commons Attribution License 4.0, which permits unrestricted use, distribution, and reproduction in any medium, provided the original work is properly cited. 